\title{
Anti-angiogenic and Anti-metastatic Effects of $\beta$-1,3-D-Glucan Purified from Hanabiratake, Sparassis crispa
}

\author{
Kyosuke Yamamoto, ${ }^{*}, a$ Takashi Kimura, ${ }^{a}$ Akio Sugitachi, ${ }^{b}$ and Nariaki Matsuura ${ }^{c}$ \\ ${ }^{a}$ Research \& Development Center, Unitika Ltd.; 23 Uji-Kozakura, Uji, Kyoto 611-0021, Japan: ${ }^{b}$ Takamatsu Hospital; 4-8 \\ Tatemukai-cho, Morioka, Iwate 020-0115, Japan: and ${ }^{c}$ School of Allied Health Sciences, Faculty of Medicine, Osaka \\ University; 1-7 Yamadaoka, Suita, Osaka 565-0871, Japan. \\ Received August 25, 2008; accepted November 12, 2008; published online November 19, 2008
}

\begin{abstract}
Sparassis crispa (SC), Hanabiratake in Japanese, is an edible mushroom with medicinal properties, that contains more than $40 \% \beta$-D-glucan. It was concluded from results of the methylation study that $\beta$-D-glucan from SC (SBG) was composed of a backbone of $\beta$ - $(1 \rightarrow 3)$-linked D-glucopyranosyl residues, and had $\beta$-D-glucopyranosyl groups joined through $O-6$ and $O-2$ of D-glucose of the backbone. We purified SBG and investigated its anti-angiogenic functions and anti-metastatic effects on neoplasm using different animal models. The oral administration of the purified SBG suppressed B16-F10 cell-induced angiogenesis in the dorsal air sac assay using female ICR mice as well as vascular endothelial growth factor induced neovascularization in the Matrigel plug assay using female C57BL/6J mice. Furthermore, it suppressed the growth and numbers of the metastatic tumor foci in lung, along with the primary tumor growth in the spontaneous metastatic model using female C57BL/6J mice. From these results, it is apparent that the oral administration of SBG results in suppressive effect on tumor growth and metastasis in lung through the inhibition of tumor induced-angiogenesis. These effects are not a result of direct action on the endothelial cells because cell growth, migration and capillary-like tube formation were not affected in the human umbilical vein endothelial cells by SBG application. This is the first report showing that the oral administration of SBG is capable of suppressing angiogenesis and metastasis.
\end{abstract}

Key words Sparassis crispa; $\beta$-( $1 \rightarrow 3)$-D-glucan; anti-angiogenesis; anti-metastasis

Sparassis crispa (SC), Hanabiratake in Japanese, is an edible mushroom with medicinal properties, which has recently become cultivable in Japan. We have already demonstrated the antitumor and antiallergic activities of this mushroom. ${ }^{1)}$ SC contains more than $40 \%$ of $\beta$-D-glucan and it has been reported that its major structural unit are $\beta$-( $1 \rightarrow 3)$-D-glucan backbone with single $\beta$-( $(1 \rightarrow 6)$-D-glucosyl side branching units every three residues. ${ }^{1-3)}$ However, a detailed structure study of $\beta$-D-glucan from SC has not been achieved yet by chemical analysis.

$\beta$-1,3-D-Glucan is a well-documented biological response modifier, ${ }^{4}$ and $\beta$-D-glucan extracted from SC (SBG) has been reported to possess many biological activities, such as antitumor effects, ${ }^{1,2}$ antiallergic effects, ${ }^{1}$ improvement of natural killer (NK) cell activity, ${ }^{1)}$ cytokine-inducing activities in the splenocytes of the mice and human peripheral blood mononuclear cells (PBMC) ${ }^{5-7)}$ and enhancement of hematopoietic responses. ${ }^{2,8-10)}$ Furthermore, a recent study has suggested the possibility that the application of SBG in cancer patients could be an effective treatment strategy. ${ }^{11)}$ However, besides immunoenhancing activities, the mechanisms of antitumor activities of $\beta$-1,3-D-glucan derived from $\mathrm{SC}$ have not been investigated thus far.

Angiogenesis is involved not only in the physiological processes such as embryonic development, ovulation and wound healing, but also in many pathological conditions such as solid tumor growth, diabetical retinopathy, age-related maculopathy and rheumatoid arthritis. ${ }^{12-15}$ ) Vascular endothelial growth factor (VEGF) is the most prominent angiogenic protein often secreted by the solid tumor cells especially under hypoxic conditions. ${ }^{16-18)}$ Newly formed blood vessels help in tumor progression and promote metastatic spread of the tumor cells. Therefore, anti-angiogenic strategies are considered to be highly effective in cancer ther- apy. ${ }^{16-18)}$

In the present study, we elucidated the primary structure of SBG using methylation analysis, investigated the anti-angiogenic effects of SBG by using two different animal models, and further assessed the anti-metastatic activities in vivo. We further analyzed the possible mechanisms of SBG function by using human umbilical vein endothelial cells (HUVECs) in vitro.

\section{MATERIALS AND METHODS}

Materials Dulbecco's modified Eagle's medium (DMEM) was obtained from Nacalai Tesque (Kyoto, Japan). HumediaEG2 medium was purchased from Kurabo (Osaka, Japan). Antibiotic-antimycotic solutions $(100 \times)$ containing 10000 units $/ \mathrm{ml}$ penicillin, $10 \mathrm{mg} / \mathrm{ml}$ streptomycin and $25 \mu \mathrm{g} / \mathrm{ml} \mathrm{am}-$ photericin B in phosphate-buffered saline (PBS) was purchased from Wako (Osaka, Japan). Fetal bovine serum (FBS) was purchased from Gibco BRL (Auckland, New Zealand). Diffusion chamber ring, MF cement and $13 \mathrm{~mm}$ circular membrane filters were obtained from Millipore (Tokyo, Japan). Growth factor reduced phenol red-free Matrigel was obtained from Becton Dickinson \& Co. (Franklin Lakes, NJ, U.S.A.). Recombinant mouse VEGF was purchased from Sigma (St. Louis, MO, U.S.A.).

Cells The highly metastatic B16-F10 and B16-BL6 cells were maintained in DMEM supplemented with $10 \%(\mathrm{v} / \mathrm{v})$ FBS, $1 \times$ antibiotic-antimycotic. HUVECs were obtained from Kurabo and cultured in HuMedia-EG2 medium supplemented with $2 \%$ (v/v) FBS, $10 \mathrm{ng} / \mathrm{ml}$ human epidermal growth factor, $1 \mu \mathrm{g} / \mathrm{ml}$ hydrocortisone, $5 \mathrm{ng} / \mathrm{ml}$ human basic fibroblast growth factor, $10 \mu \mathrm{g} / \mathrm{ml}$ heparin, $50 \mu \mathrm{g} / \mathrm{ml}$ gentamicin and $50 \mathrm{ng} / \mathrm{ml}$ amphotericin B.

Animals Female ICR (4 weeks old) and C57BL/6J mice 
(5 weeks old) were obtained from Japan SLC (Shizuoka, Japan), and Clea Japan (Osaka, Japan), respectively. They were housed for 1 week in a room maintained at $24 \pm 1^{\circ} \mathrm{C}$ with a $12 \mathrm{~h}$ light-dark cycle with free access to diet (Labo MR Stock, Nihon Nosan Kogyo) and water. The mice were treated according to the ethical guidelines prescribed by the Animal Study of Unitika Ltd.

Purification of $\boldsymbol{\beta}$-Glucan from SC (SBG) $\quad \beta$-Glucan purified from SC was named SBG. SBG was purified enzymatically from the fruiting bodies of SC cultivated by Unitika Ltd. Briefly, powdered SC was suspended in $0.08 \mathrm{M}$ phosphate buffer ( $\mathrm{pH}$ 6.0) and treated with thermostable $\alpha$-amylase (Sigma) for $30 \mathrm{~min}$ in boiling water. Then, subtilisin A (Sigma) treatment $\left(30 \mathrm{~min}, 60^{\circ} \mathrm{C}, \mathrm{pH} 7.5\right)$ and amyloglucosidase (Sigma) $\left(30 \mathrm{~min}, 60^{\circ} \mathrm{C}, \mathrm{pH} 4.3\right)$ treatment was carried out in succession, followed by $80 \%(\mathrm{v} / \mathrm{v})$ ethanol precipitation. The precipitate was resuspended in water, and dialyzed against deionized water. Inner solution was again precipitated with ethanol (final conc. $80 \%(\mathrm{v} / \mathrm{v})$ ) and dried under reduced pressure. The final yield of precipitates was $65.0 \%$, and the total sugar content was measured as $98.2 \%$ using the phenolsulfuric acid method with glucose as the standard. ${ }^{19)}$

Metylation Analysis of SBG $2.7 \mathrm{mg}$ of SBG was methylated by Hakomori procedure. ${ }^{20)}$ The product was confirmed to show no absorption for the hydroxyl group in its IR spectrum, and it hydrolyzed with $90 \%$ formic acid and then with $0.5 \mathrm{~m}$ sulfuric acid. The partially methylated sugar thus obtained was converted to the alditol acetate ${ }^{21)}$ for GC-MS analyses. Gas chromatograph-mass spectrometer was conducted with a JMS DX-303 apparatus equipped with a fused silica capillary column SPB-5 (Supelco Japan) $(0.25 \mathrm{~mm} \times$ $30 \mathrm{~m}$ ) programmed from 60 to $280{ }^{\circ} \mathrm{C}$ at $8^{\circ} \mathrm{C} / \mathrm{min}$ with a gas flow rate of $50 \mathrm{ml}$ of $\mathrm{He}$ per min for partially methylated alditol acetate, and the spectra were recorded at an ionizing potential of $70 \mathrm{eV}$.

Tumor-Induced Angiogenesis in the Dorsal Air Sac (DAS) Assay DAS assay was carried out according to the method described by Oikawa et $a .^{22)}$ with slight modification. Briefly, both sides of the diffusion chamber ring were covered with membrane filters, and the resulting chambers were filled with B16-F10 cells $\left(2 \times 10^{6}\right.$ cells $)$ in $150 \mu \mathrm{l}$ of PBS. Each B16-F10 containing chamber was implanted into the DAS of female ICR mice on day 0 . The negative control group was implanted with PBS containing chamber. SBG was orally administered once a day from $6 \mathrm{~d}$ before to $6 \mathrm{~d}$ after the day 0 for a total of $13 \mathrm{~d}$. On day 7, each mouse was sacrificed and tumor cell-induced angiogenesis at the implanted zone was observed. The number of newly formed blood vessels $>3 \mathrm{~mm}$ in length with a characteristic zigzag shape was counted.

VEGF-Induced Angiogenesis in the Matrigel Plug Assay The Matrigel plug assay was performed according to the method described by Kimura et al. ${ }^{23)}$ Briefly, each female C57BL/6J mice was subcutaneously injected with $0.5 \mathrm{ml}$ of Matrigel containing $20 \mathrm{ng} / \mathrm{ml} \mathrm{VEGF}$ and $32 \mathrm{U} / \mathrm{ml}$ heparin on day 0 . Negative control group was injected with Matrigel alone. SBG was orally administered once a day from $7 \mathrm{~d}$ before to $5 \mathrm{~d}$ after the day 0 . On day 6 , each Matrigel was excised and weighed, and then the gel was treated with dispase-II (1.5 mg/ml, Roche Diagnostics, Tokyo, Japan) followed by determination of hemoglobin content using the Quantichrom hemoglobin assay kit (Funakoshi, Tokyo, Japan).

Tumor Growth and Spontaneous Lung Metastasis Spontaneous pulmonary metastasis assay was carried out based on the method described by Murata et al. ${ }^{24)}$ Briefly, highly-metastatic B16-BL6 cells $\left(1.5 \times 10^{5}\right)$ in PBS were injected into the right footpad of female $\mathrm{C} 57 \mathrm{BL} / 6 \mathrm{~J}$ mice on day 0 . SBG was orally administered six times per week from $7 \mathrm{~d}$ before to $44 \mathrm{~d}$ after the day 0 . Tumor growth was measured every 3-4 d from day 11 to day 24 and indicated as volume, which was calculated using the following formula: tumor volume $\left(\mathrm{mm}^{3}\right)=\pi \times($ large diameter $) \times(\text { small diameter })^{2} /$ 6 . To examine lung metastasis, primary tumors were excised on day 24 and the mice were kept alive for another 3 weeks. On day 45, lungs were removed and the number and size of pulmonary metastatic colonies was counted using a stereomicroscope.

In Vitro Proliferation, Migration and Capillary Morphogenesis of HUVECs To assess the proliferation of HUVECs, the cells were seeded into a 96-multiwell culture plate at $5 \times 10^{3}$ cells $/ \mathrm{ml}$ and SBG was added. Proliferation of the HUVECs was measured using the cell counting kit (CCK-8; Dojindo laboratories, Kumamoto, Japan) at 24 and $68 \mathrm{~h}$ post seeding. Migration of the HUVECs was examined by wound healing assay. ${ }^{25)}$ The HUVECs were cultured to confluency in a 24-multiwell culture plate. The monolayer was scratched using $200 \mu \mathrm{l}$ pipet tips, following which the width of the wound was measured after $9 \mathrm{~h}$. Capillary morphogenesis was observed using two-dimensional Matrigel-based assay, as described by Stanley et $a l^{26)}$ Briefly, $0.2 \mathrm{ml}$ of Matrigel was coated onto a 24-multiwell plate, The HUVECs were seeded at a density of $4 \times 10^{4}$ cells/well, followed by the addition of SBG. Sixteen hours post seeding, capillary morphogenesis was observed using a microscope. Quantification of the capillary tube formation was performed by counting the number of tubule junctions.

Statistical Analysis All data were represented as mean \pm S.E. Differences among means were analyzed by the Student's $t$-test or Mann-Whitney $U$-test. Differences were considered significant at $p<0.05$.

\section{RESULTS}

Primary Structure of SBG GLC gave four peaks, and each was identified by its mass spectrum. These are summarized in Table 1 . These results suggested that the major structural units of SBG are a $\beta$ - $(1 \rightarrow 3)$ - D-glucan backbone with two $\beta$-( $(1 \rightarrow 6)$-D-glucosyl side and single $\beta$-( $1 \rightarrow 2)$-D-glucosyl side branching units every ten residues, as shown in Fig. 1.

Effects of Oral Administration of SBG on Tumor Induced Angiogenesis We examined the effects of SBG on tumor-induced neovascularization using the DAS system. Angiogenesis was strongly induced after implantation of B16-F10 cells in the chamber; however, oral administration of SBG significantly inhibited the tumor-induced angiogenesis (Fig. 2).

Effects of Oral Administration of SBG on VEGF-Induced Angiogenesis We investigated the anti-angiogenic effects of SBG using another in vivo model of angiogenesis, the Matrigel plug assay. Remarkable increase in the Matrigel weight and the hemoglobin content was observed in the group injected with Matrigel containing VEGF compared to 
Table 1. Summary of Methylation Analysis of SBG

\begin{tabular}{lccc}
\hline \hline \multicolumn{1}{c}{ Bond } & Feature & Ratio $^{a)}$ & Ratio (\%) \\
\hline Glc $1 \rightarrow$ & Non-reducing end & 1.00 & 21.9 \\
$\rightarrow 3$ Glc $1 \rightarrow$ & $\beta$-1,3-Bond & 2.48 & 54.4 \\
$\rightarrow 2,3$ Glc $1 \rightarrow$ & Branching site & 0.37 & 8.4 \\
$\rightarrow 3,6$ Glc $1 \rightarrow$ & Branching site & 0.71 & 15.6 \\
\hline Total & & 4.56 & 100.0 \\
\hline
\end{tabular}

a) Alditol acetate of 2,3,4,6-tetra- $O$-methyl glucose (Glc $1 \rightarrow$ ) was adjusted to 1.00 .

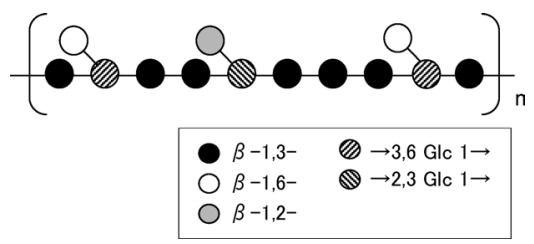

Fig. 1. Structure Model of SBG Presumed by Methylation Analysis

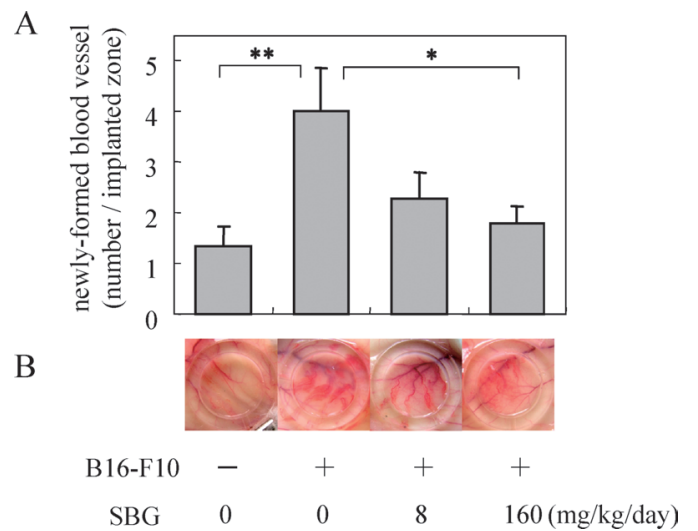

Fig. 2. Effects of SBG on Tumor-Induced Angiogenesis in the DAS Assay System

The number of newly formed blood vessels $>3 \mathrm{~mm}$ length with a characteristic zigzag shape in the chamber implanted zone was indicated (A). Representative photographs at the chamber-implanted zone of each group (B). $* p<0.05$, $* * p<0.01 . n=6-9$.

the group treated with Matrigel alone. Conversely, such angiogenic responses were significantly suppressed by oral administration of SBG (Fig. 3).

Effects of Oral Administration of SBG on Tumor Growth and Lung Metastasis Anti-angiogenic therapy is well-accepted in recent cancer therapy since angiogenesis is an essential event involved in tumor progression and metastasis. We investigated the effect of SBG on tumor growth and lung metastasis. Tumor growth rate of subcutaneously injected B16-BL6 cells was retarded by the oral administration of SBG (Fig. 4). Furthermore, the number as well as the size of the pulmonary metastatic tumor colonies was significantly reduced by SBG administration (Fig. 5).

Effects of SBG on Proliferation, Migration, and Capillary Morphogenesis of HUVECs To investigate possible mechanisms of SBG action against neovascularization, we examined the effect of SBG on HUVECs in vitro. The cells were treated with SBG and assessed for proliferation, migration, and capillary tube formation. Despite high dose application of SBG, none of the above mentioned properties of HUVECs were affected (Fig. 6).
A

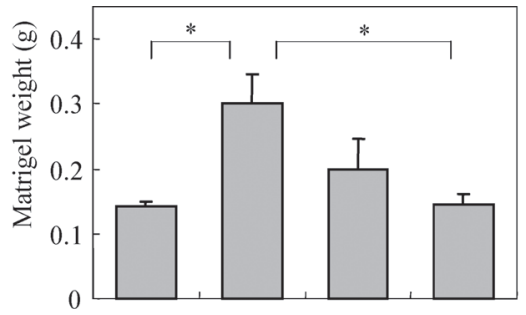

B

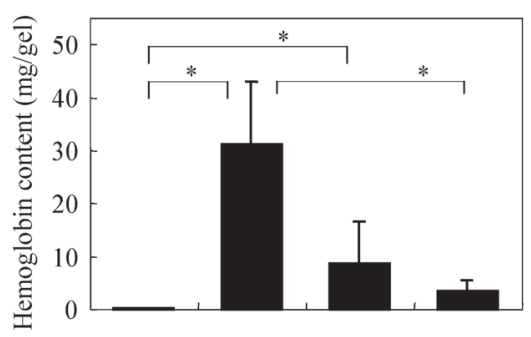

C

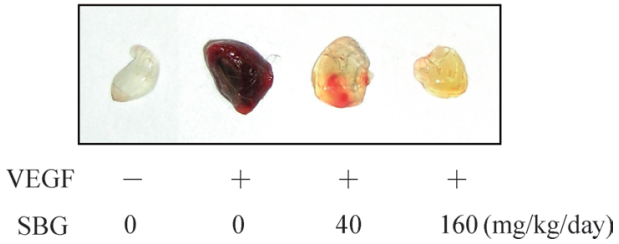

Fig. 3. Effects of SBG on VEGF-Induced Angiogenesis in Matrigel Plug Assay

Matrigel weight (A), hemoglobin content in the Matrigel (B), representative photographs of excised Matrigels of each group (C). * $p<0.05 . n=5-6$.



Fig. 4. Effects of SBG on Primary Tumor Progression

$* p<0.05, * * p<0.01$ (vs. control group at each time point). $n=8-10$.

\section{DISCUSSION}

SC (Hanabiratake in Japanese), is an edible and medicinal mushroom containing large amount of $\beta$-1,3-D-glucan. SC is known to show antitumor activity in oral administration. ${ }^{1)} \beta$ 1,3-D-glucan of SC is reported to act as an antitumor agent in tumor-bearing mice when injected intraperitoneally and a stimulator of hematopoiesis on intraperitoneal and oral administration in vivo. ${ }^{2,8-10)}$ Moreover, the glucan also enhances the cytokine production in mice spleen cells and human PBMC. ${ }^{5-7)}$

It is well accepted that the biological effects of $\beta$-D-glucan depend on its primary structures. Although SBG was struc- 
A

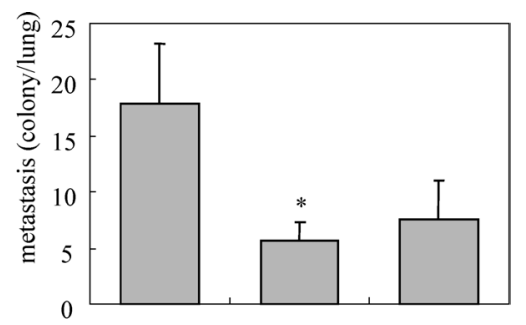

$\mathrm{B}$

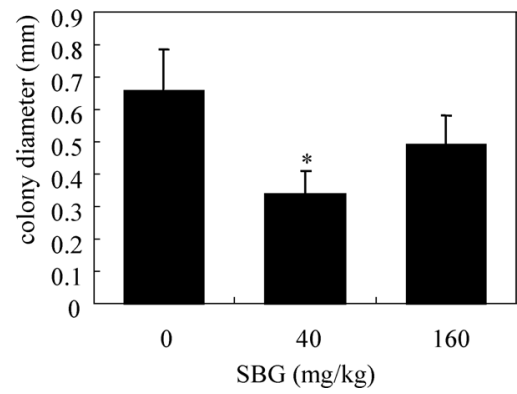

Fig. 5. Effects of SBG on Spontaneous Metastasis

The number of pulmonary metastatic colony (A), size of metastatic foci (B). $* p<0.05$ (vs. control group). $n=6-7$.

A

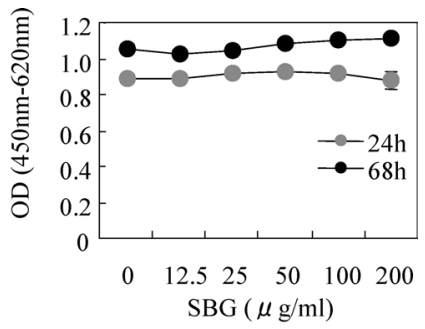

B

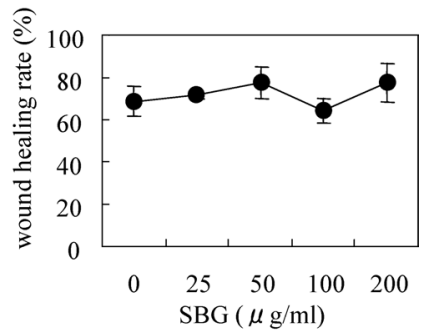

$\mathrm{C}$

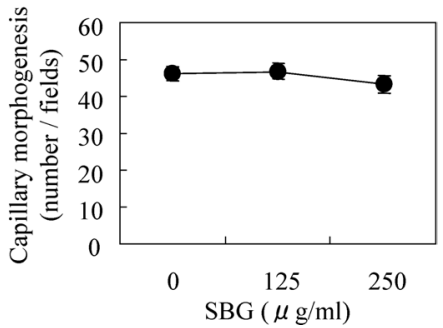

Fig. 6. Effects of SBG on HUVECs

Proliferation (A), wound healing rate (B) and capillary morphogenesis (C). $n=3-8$.

turally the same as $\beta$-D-glucan from Schizophyllum commune, $\mathrm{SPG}$, which major structural unit is $\beta$-(1 $\rightarrow 3)$-D-glucan backbone with single $\beta$-(1 $\rightarrow 6)$-D-glucosyl side branching units every three residues, strangely, SBG but not SPG induce cytokine production from bone marrow-derived dendritic cells (BMDCs). ${ }^{3)}$

Hence, we tried to elucidate the primary structure of SBG by chemical analysis, and found $\beta-(1 \rightarrow 2)$-D-glucosyl side branching units newly, except for $\beta$ - $(1 \rightarrow 6)$-D-glucosyl side branching units. The difference of the biological activity between SBG and SPG might be derived from the existence $\beta$ $(1 \rightarrow 2)$-D-glucosyl side branching units.

The antitumor mechanisms of SC, except for the immunomodulating actions, have not been well studied. Hence, we tried to elucidate possible mechanisms of anti-angiogenic potential of SBG using two different animal models. In this study, we investigated the effect of SBG on angiogenesis, particularly by oral administration, since there are very few reports demonstrating that orally administered glucan inhibits neovascularization.

First, we demonstrated the anti-angiogenic activity of SBG in the DAS system. The suppressive effect of VEGF-induced neovascularization was further confirmed in Matrigel plug assay which enables sustained release of VEGF in vivo. We further analyzed the anti-metastatic effects of SBG, since angiogenesis is an essential event for tumor cells to grow in vivo and to spread by metastasis. In B16-BL6-bearing mice, tumor growth rate was significantly retarded by preventive administration of SBG. And the number of lung metastatic colonies and growth of these metastatic foci was also significantly suppressed. In this experiment, dose-dependency of anti-metastatic effects was not observed, however, Miura et $a l^{27)}$ has reported that overdose application of $\beta$-glucan (sonifilan) fails in expressing antitumor activity. Hence, appropriate dosage should be essential for long-term use of SBG.

In all animal experiments in this study, SBG was pre-administered before surgical treatment of mice to estimate significant effects of SBG. We did not evaluate the effects of SBG without pre-administration. However, in our previous study of powdered-SC using tumor-bearing mice, post-administration after tumor injection was effective to suppress tumor growth.

Several mushrooms have been reported to show anti-angiogenic and anti-metastatic effects, such as Agaricus blazei, Ganoderma lucidum, Cordyceps militaris, and Coriolus vesicolor, and their active substances have been identified as triterpenoid, ergosterol, and pyroglutamate etc. $23,25,26,28-30)$ Fungal protein-bound polysaccharide derived from C. vesicolor (PSK) was also reported to act as an anti-angiogenic or anti-metastatic agent. ${ }^{31)}$ However, PSK is mainly constituted of $\beta$-1,4-bond glucan main chain having $\beta-1,3$ and $\beta-1,6$ bond side chain binding to a protein moiety. ${ }^{32)}$ Therefore, it was considered that SBG is a novel anti-angiogenic and antimetastatic agent being particularly effective in oral administration.

The mechanisms of anti-angiogenic actions of SBG as well as PSK remain to be clarified; the possibilities include inhibitory effects of proliferation, migration and capillary morphogenesis of vascular endothelial cells. We therefore examined the direct actions of SBG on HUVECs, however, no significant effects were shown. We have not examined the metabolism of SBG, however, it is reported that $98.4 \%$ of orally-administered $\beta$-glucan (SSG from Sclerotinia sclerotiorum) were excreted with the feces. ${ }^{33)}$ And also, $\beta$-glucan is a kind of dietary fiber and highly resistant against digestive enzymes. Therefore, metabolite of $\beta$-glucan is not likely to contribute these effects directly. These observations suggest that SBG suppresses angiogenesis of vascular endothelial 


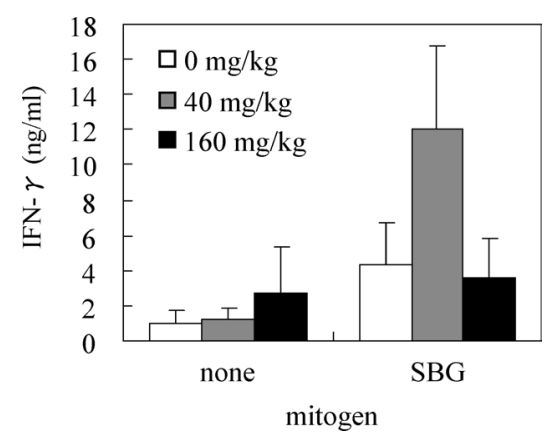

Fig. 7. IFN- $\gamma$ Secretion from Splenic Lymphocytes Derived from SBGFed Mice

Splenic lymphocytes were cultured with or without $100 \mu \mathrm{g} / \mathrm{ml} \mathrm{SBG}$ for $72 \mathrm{~h} . n=4$.

cells by an indirect mode of action.

$\beta-1,3-\mathrm{D}-$ Glucan derived from $\mathrm{SC}$ is reported to stimulate interferon (IFN)- $\gamma$ and IL-12p70 production from mice spleen cells. ${ }^{4,5)}$ Several cytokines such as tumor necrosis factor (TNF)- $\alpha$, IFN- $\alpha$, IFN- $\gamma$, IL-12, and interferon-inducible protein 10 (IP-10) are known anti-angiogenic factors. ${ }^{34-37)}$ In the present study, it might be possible that such anti-angiogenic proteins contribute to the action of SBG because IFN- $\gamma$ production from splenic lymphocytes of SBG-fed mice was relatively increased compared to the control group (Fig. 7). When lymphocytes were stimulated by SBG, IFN- $\gamma$ secretion level was the highest in $40 \mathrm{mg} / \mathrm{kg} / \mathrm{d}$ group. It was correlated with the inhibition activity of metastasis. The possibility that orally administered SBG leads to enhanced production of anti-angiogenic proteins needs further investigation.

In conclusion, our results indicate that oral administration of $\beta$-D-glucan purified from SC, which has $\beta$ - $(1 \rightarrow 6)$ and $\beta$ $(1 \rightarrow 2)$-D-glucosyl side branching units, showed anti-angiogenic and anti-metastatic effects in mice. Our observations suggest that the anti-angiogenic effect of $\beta$-glucan purified from SC may be involved in the anti-metastatic effects as well as antitumor effects.

Acknowledgement We thank Dr. Hiromi Ashino in The Tokyo Metropolitan Institute of Medical Science (RINSHOKEN) for her technical suggestions.

\section{REFERENCES}

1) Hasegawa A., Yamada M., Dombo M., Fukushima R., Matsuura N., Sugitachi A., Jpn. J. Cancer Chemother, 31, 1761-1763 (2004).

2) Ohno N., Miura N. N., Nakajima M., Yadomae T., Biol. Pharm. Bull., 23, 866-872 (2000)

3) Tada R., Harada T., Nagi-Miura N., Adachi Y., Nakajima M., Yadomae T., Ohno N., Carbohydr. Res., 342, 2611-2618 (2007).

4) Mohagheghpour N., Dawson M., Hobbs P., Judd A., Winant R., Dousman L., Waldeck N., Hokama L., Tuse D., Kos F., Benike C., Engleman E., Adv. Exp. Med. Biol., 383, 13-22 (1995).
5) Harada T., Miura N. N, Adachi Y., Nakajima M., Yadomae T., Ohno N., J. Interferon Cytokine Res., 22, 1227-1239 (2002).

6) Harada T., Miura N. N., Adachi Y., Nakajima M., Yadomae T., Ohno N., J. Interferon Cytokine Res., 24, 478-489 (2004).

7) Nameda S., Harada T., Miura N. N., Adachi Y., Yadomae T., Nakajima M., Ohno N., Immunopharmacol. Immunotoxicol., 25, 321-335 (2003).

8) Harada T., Miura N. N, Adachi Y., Nakajima M., Yadomae T., Ohno N., Biol. Pharm. Bull., 25, 931-939 (2002).

9) Ohno N., Harada T., Masuzawa S., Miura N. N., Adachi Y., Nakajima M., Yadomae T., Int. J. Med. Mushr., 4, 13-26 (2002).

10) Harada T., Kawaminami H., Miura N. N., Adachi Y., Nakajima M., Yadomae T., Ohno N., Microbiol. Immunol., 50, 687-700 (2006).

11) Ohno N., Nameda S., Harada T., Miura N. N., Adachi Y., Nakajima M., Yoshida K., Yoshida H., Yadomae T., Int. J. Med. Mushr., 5, 359368 (2003).

12) Folkman J., Nat. Med., 1, 27-31 (1995).

13) Martiny-Baron G., Marmé D., Curr. Opin. Biotechnol., 6, 675-680 (1995).

14) Carmeliet P., Jain R. K., Nature (London), 407, 249-257 (2000).

15) Yamauchi M., Ohmi S. I., Shibuya M., Cancer Sci., 98, 1491-1497 (2007).

16) Liao D., Johnson R. S., Cancer Metastasis Rev., 26, 281 -290 (2007).

17) Tsunoda S., Nakamura T., Sakurai H., Saiki I., Cancer Sci., 98, 541548 (2007).

18) Keedy V. L., Sandlar A. B., Cancer Sci., 98, 1825-1830 (2007).

19) Dubois M., Gilles K. A., Hamilton J. K., Robers P. A., Smith F., Anal. Chem., 28, 350-356 (1956).

20) Hakomori S., J. Biochem., 55, 205-208 (1964).

21) Blakeney A. B., Harris P. J., Henry R. J., Stone B. A., Carbohydr. Res., 113, 291-299 (1993).

22) Oikawa T., Sasaki M., Inose M., Shimamura M., Kuboki H., Hirano S., Kumagai H., Ishizuka M., Takeuchi T., Anticancer Res., 17, 18811886 (1997).

23) Kimura Y., Kido T., Takaku T., Sumiyoshi M., Baba K., Cancer Sci., 95, 758-764 (2004).

24) Murata J., Saiki I., Nishimura S., Nishi N., Tokura N., Azuma I., Jpn. J. Cancer Res., 80, 866-872 (1989).

25) Yoo H.-S., Shin J.-W., Cho J.-H., Son C.-G., Lee Y.-W., Park S.-Y., Cho C.-K., Acta Pharmacol. Sin., 25, 657-665 (2004).

26) Stanley G., Harvey K., Slivova V., Jiang J., Sliva D., Biochem. Biophys. Res. Commun., 330, 46-52 (2005).

27) Miura T., Miura N. N., Ohno N., Adachi Y., Shimada S., Yadomae T., Biol. Pharm. Bull., 23, 249-253 (2000).

28) Takaku T., Kimura Y., Okuda H., J. Nutr., 131, 1409-1413 (2001).

29) Kimura Y., Taniguchi M., Baba K., Anticancer Res., 22, 3309-3318 (2002).

30) Ho J. C. K., Konerding M. A., Gaumann A., Groth M., Liu W. K., Life Sci., 75, 1343-1356 (2004).

31) Kanoh T., Matsunaga K., Saito K., Fujii T., In Vivo, 8, 247-250 (1994).

32) Kobayashi H., Matsunaga K., Oguchi Y., Cancer Epidemiol. Biomarkers Prev., 4, 275-281 (1995).

33) Ohno N.: 〈http://www.dojindo.co.jp/dojinnews/2006.html〉, Dojin News, 114, 1-10 (2005).

34) Fajardo L. F., Kwan H. H., Kowalski J., Prionas S. D., Allison A. C., Am. J. Pathol., 140, 539-544 (1992).

35) Maheshwari R. K., Srikantan V., Bhartiya D., Kleinman H. K., Grant D. S., J. Cell Physiol., 146, $164-169$ (1991).

36) Rüegg C., Yilmaz A., Bieler G., Bamat J., Chaubert P., Lejeune F. J., Nat. Med., 4, 408-414 (1998).

37) Sgadari C., Angiolillo A. L., Tosato G., Blood, 87, 3877-3882 (1996). 